\title{
Gestión universitaria latinoamericana durante la pandemia
}

\section{Latin American university management during the pandemic}

DOI: $10.46932 / s f j d v 2 n 5-128$

Received in: Oct 1st, 2021

Accepted in: Dec 30th, 2021

\section{Carlos Rios-Campos}

Investigador RENACYT. Doctor en Gestión Universitaria. Maestro en Administración. Ingeniero de Sistemas. Docente de la Universidad Nacional Toribio Rodríguez de Mendoza de Amazonas. Miembro del Colegio de Ingenieros del Perú. Section Investigation Activities Chair North (IAC) - IEEE Perú.

Dirección: Calle La Plata 155 - Urb: San Eduardo - Chiclayo, Perú.

E-mail: carlos.rios@untrm.edu.pe

\section{Nemesio Santamaría Baldera}

Maestro en Administración Educativa y Desarrollo Sostenible. Licenciado en Física. Presidente de la Comisión de Admisión. Docente auxiliar de la Universidad Nacional Intercultural Fabiola Salazar Leguía de Bagua. Dirección: Ancash 520 - Bagua, Perú.

E-mail: nsantamaria@unibagua.edu.pe

\section{Juan Alberto Avalos Hubeck}

Maestro en Tributación y Asesoría Fiscal. Contador Público. Docente de la Universidad Nacional Toribio Rodríguez de Mendoza de Amazonas. Dirección: Olimpo 129 - Urbanización El Paraíso -

Chiclayo, Perú.

E-mail: juanavalosh@hotmail.com

\section{Janet Avalos Hubeck}

Mg Gestion de Servicios de Salud. Lic en Enfermeria. Docente de la Universidad Señor de Sipán. Dirección: Olimpo 129 - Urbanización El Paraíso - Chiclayo, Perú.

E-mail: javalosh@crece.uss.edu.pe

\section{Enrique Guillermo Llontop Ynga}

Médico Cirujano. Magister en Gestión de Servicios de Salud, Universidad Señor de Sipán, Universidad Cesar Vallejo. Dirección: Olimpo 129 - Urbanización El Paraíso - Chiclayo, Perú.

E-mail: ellontop@crece.uss.edu.pe/ ellontopy@ucvvirtual.edu.pe

\section{Daniel Jesús Castro Vargas}

Magister en Administración de la Educación. Licenciado Administración de Empresas. Contador Público Colegiado. Director del Instituto de Investigación de Desarrollo Social de la Universidad Nacional Autónoma de Chota - UNACH. Dirección: Diego Palomino Nº 1618 - Jaén - Cajamarca, Perú.

E-mail: djcastrov@unach.edu.pe

\section{Giuliana Fiorella Samame Aguirre}

Médico cirujano, Especialista en Anestesiología. Maestra en Gestión de los Servicios de Salud, Maestra en Medicina con Mención en Cirugía. Hospital Leoncio Prado. Dirección: Av 10 de julio 209 Huamachuco, Perú.

E-mail: giulianasamame31@gmail.com 


\title{
Juan Alberto Maza Riojas
}

Maestría con mención en Gerencia Educativa Estratégica. Licenciado en Educación. Docente de la I.E. N 15489 - "Minas De Trigal" - Huarmaca. Dirección: Avenida Sucre 960 - Caserío Pativilca, Distrito de Pitipo, Provincia de Ferreñafe, Departamento de Lambayeque, Perú.

E-mail: jmazariojas25@gmail.com

\section{RESUMEN}

Es necesario comprender lo sucedido en las universitarias latinoamericanas, considerando que a nivel mundial se presentó un desafío con el inicio de la pandemia en el año 2020. Se planteó el objetivo general, analizar la gestión universitaria latinoamericana durante la pandemia. Metodología, la investigación presentó un diseño cualitativo-interpretativo, de tipo documental, se han seleccionado 59 documentos, realizados en el periodo 2016 - 2021, incluyendo: artículos científicos, artículos de revisión e información de sitios web de organizaciones reconocidas. Las palabras claves utilizadas en las búsquedas fueron: Gestión universitaria, Latinoamérica, pandemia. Resultados, las universidades latinoamericanas, se han visto forzadas por la pandemia a migrar a la educación virtual, con algunas dificultades como la falta de equipos tecnológicos, acceso a Internet y capacitación del personal. Además, están optimizando su modelo de gestión universitaria. Conclusiones, en las universidades latinoamericanas, los factores con mayor influencia en la gestión universitaria actual son la infraestructura, la gestión de la información, inversión en sistemas de información, capacitación de los recursos humanos, empleabilidad del egresado, calidad de los procesos académicos, científicos y administrativos, responsabilidad social, competencias en el área de educación virtual, rol protagónico en la gestión de la pandemia, gestión de riesgos de desastres y el regreso a la presencialidad.

Palabras clave: Gestión universitaria, Latinoamérica, pandemia.

\begin{abstract}
It is necessary to understand what happened in Latin American universities, considering that a global challenge was presented with the onset of the pandemic in the year 2020. The general objective was to analyze Latin American university management during the pandemic. Methodology, the research presented a qualitative-interpretative design, of documentary type, 59 documents were selected, carried out in the period 2016 - 2021, including: scientific articles, review articles and information from websites of recognized organizations. The keywords used in the searches were: university management, Latin America, pandemic. Results, Latin American universities, have been forced by the pandemic to migrate to virtual education, with some difficulties such as lack of technological equipment, Internet access and staff training. In addition, they are optimizing their university management model. Conclusions: In Latin American universities, the factors with the greatest influence on current university management are infrastructure, information management, investment in information systems, human resources training, graduate employability, quality of academic, scientific and administrative processes, social responsibility, competencies in the area of virtual education, leading role in the management of the pandemic, disaster risk management, and the return to the face-to-face mode.
\end{abstract}

Keywords: University management, Latin America, pandemic.

\section{INTRODUCCIÓN}

En esta investigación se planteó el objetivo general, analizar la gestión universitaria latinoamericana durante la pandemia. 
En su condición de único organismo de las Naciones Unidas que ha recibido un mandato relativo a la educación superior, la UNESCO apoya los esfuerzos que los países llevan a cabo para mejorar la calidad y garantizar las prestaciones en materia de educación superior, con miras a fomentar la equidad y propiciar un mejor reparto de oportunidades entre todos los estudiantes (UNESCO, 2021).

\subsection{LATINOAMÉRICA}

América Latina y el Caribe es una región geopolítica que comprende más de 40 países y territorios desde México hasta el Cabo de Hornos, y que puede subdividirse en cuatro regiones en función de su ubicación geográfica: América del Sur, América Central, el Caribe y México (Statista Research Department, 2021).

La ubicación de América Latina y el Caribe en la nueva geografía del desarrollo resulta ambigua: es una región en la que predominan las rentas medias y que, aun así, apenas alberga a un 3\% de la población pobre mundial (Tezanos, 2018).

Los países de América Latina y el Caribe, a través de la Resolución 700 (XXXVI) aprobada en mayo del 2016 en el marco del Trigésimo Sexto Periodo de Sesiones de la Comisión Económica para América Latina y el Caribe (CEPAL), crearon el Foro de los Países de América Latina y el Caribe sobre el Desarrollo Sostenible como mecanismo regional para la implementación y seguimiento de la Agenda 2030 para el Desarrollo Sostenible, los ODS, sus metas y sus medios de implementación, así como para la Agenda de Acción de Addis Abeba sobre el Financiamiento para el Desarrollo (CEPAL, 2021a).

\subsection{PANDEMIA}

América Latina es la región más golpeada por la pandemia de COVID-19. La fuerte contracción producto de la crisis sanitaria ha tenido enormes costos económicos y sociales, ya que llegó después de varios años de un débil desempeño, con un bajo crecimiento promedio y progreso limitado en los indicadores sociales, y tras un periodo de agitación social que sacudió a algunos países a finales de 2019 (Banco Mundial (2021).

La OPS colabora con los países latinoamericanos en la protección del personal sanitario, impartiendo cursos de capacitación sobre cómo evitar las infecciones y procurar equipos de protección individual. Es fundamental que los Gobiernos decidan qué medidas de distanciamiento social son necesarias aplicar y por cuánto tiempo (Naciones Unidas, 2020).

El informe destaca que la recuperación sostenida tras la pandemia dependerá no solo del alcance de las medidas de estímulo y la rápida introducción de las vacunas, sino también de la calidad y la eficacia que tengan esas medidas para aumentar la resiliencia contra las futuras crisis (CEPAL, 2021b). 
Una salud internacional sur sur que no sólo implica esperar el gobierno del Estado para lograr nuevas relaciones intergubernamentales como base para nuevos cimientos de soberanía políticosanitaria. Este camino comienza desde y con las sociedades del Sur global: entre nuestras redes, territorialidades, diálogos interculturales y relaciones transmodernas (Basile, 2020).

\subsection{GESTIÓN UNIVERSITARIA}

Dentro de la gestión universitaria es necesario evaluar el cumplimiento de la misión y fines institucionales, con un alto sentido de responsabilidad, que está íntimamente relacionado con los intereses de la sociedad, por el impacto de estas organizaciones en la trasferencia del conocimiento, costumbres y raíces culturales (Murillo, 2019).

En los tiempos actuales y futuros, en condiciones de medidas extremas, generadas por la pandemia mundial de Covid-19, se hace necesario perfeccionar en gran medida la gestión universitaria, donde los cambios en la proyección y la planificación, en este nuevo contexto, son definitivos (Gil, Morales \& Nieto, 2020).

Desde esta perspectiva, los intercambios informacionales en la gestión universitaria asumen una plataforma social y una plataforma tecnológica capaz de guiar los procesos universitarios hacia dos grandes fines: la transformación social y la canalización eficiente de dicho cambio a través del uso intensivo de las TIC y la virtualidad en contextos de aislamiento social (Corral, Izurieta \& Macías, 2020).

\section{METODOLOGÍA}

La investigación presenta un diseño cualitativo-interpretativo, de tipo documental, el cual precisó el procedimiento de selección y el registro de los datos (Barrero y Rosero, 2018).

En la presente investigación se han seleccionado 59 documentos, realizados en el periodo 2016 2021; incluyendo: artículos científicos, artículos de revisión e información de sitios web de organizaciones reconocidas. Las palabras claves utilizadas en las búsquedas fueron: Gestión universitaria, Latinoamérica, pandemia. Para la selección de los documentos se usaron como criterios: el año de la publicación, la pertenencia con la investigación y ser una fuente confiable. Después de la lectura de cada documento, se procedió a ingresar los datos a la matriz bibliográfica, que sirve para catalogar los documentos de acuerdo con categorías, que se presentan en la tabla 1.

Tabla 1. Matriz bibliográfica

\begin{tabular}{|l|l|l|l|}
\hline Nombre & Tipo & Objetivos & Conclusiones \\
\hline & & & \\
\hline & & & \\
\hline
\end{tabular}

Fuente: Adaptado de Barrero y Rosero (2018). 


\section{RESULTADOS}

Como hemos analizado en este artículo, la inducción poco a poco va contemplándose como una política educativa normalizada en muchos países de América Latina y el Caribe. Existe un consenso común en relación a los beneficios que la inducción de calidad aporta a los docentes, las escuelas y en general al sistema educativo (Marcelo y Vaillant, 2017).

Las universidades iberoamericanas han de encontrar nuevas maneras para crecer y llevar a cabo más proyectos más que eliminarlos o no hacerlos. La universidad presencial se transforma en un nuevo lugar plagado de retos y no puede perder el control teniendo que abrazar los grandes progresos del online...(Barquero-Cabrero, Barceló-Sánchez, López-Martín \& Cabezuelo-Lorenzo, 2020).

Los Coloquios Internacionales de "Gestión Universitaria - CIGU”, son un ejemplo de trabajo en conjunto y cooperación que reunieron inicialmente como organizadores a la Universidad Federal de Santa Catarina (UFSC), por intermedio del Programa de Pos-Graduación en Administración (CPGA), del Instituto de Investigación y Estudios en Administración Universitaria (INPEAU), en Brasil, y la Maestría en Gestión Universitaria de la Universidad Nacional de Mar del Plata (UNMdP), de la Asociación de Especialistas en Gestión de la Educación Superior (AEGES), constituido en el 2000 en Argentina, para despertar una nueva visión sobre la universidad en América (Oficina Universitaria de Imagen Institucional, 2019).

Las variables más influyentes en la gestión universitaria se concentran en la infraestructura y la gestión de la información; las de mayor efecto en los procesos y los recursos humanos, de manera que la generación de capacidades desarrolladas por la infraestructura se refleja en el desempeño de los procesos y estos también pasan por la gestión de la información y el desempeño de los recursos humanos (VelizBriones, Alonso-Becerra, Fleitas-Triana \& Alfonso-Robaina, 2016).

Estos hallazgos permiten a los gestores de las universidades tomar decisiones sobre los SI en los que deben invertir y apoyar su implementación. De igual manera, es importante que conozcan los resultados de aquellos análisis que no permiten establecer relación con el logro de un posicionamiento en los rankings, de manera que las universidades puedan tomarlo en consideración para concentrarse en los que sí contribuyen a posicionarse en los rankings (Gerón-Piñón, Solana-González, TriguerosPreciado \& Pérez-González, 2021).

El grado de participación del egresado en las actividades de Extensión Cultural y Proyección Social y la Facultad, como buen facilitador de las prácticas pre-profesionales, favorece la interrelación del Egresado con los demás profesionales de su entorno laboral...La forma de gestionar de las universidades influye en la empleabilidad del egresado porque eleva o reafirma las competencias del Egresado (Roque, 2018). 
Se consideran como debilidades del sistema de educación superior en América Latina, la calidad del sistema de educación superior y la calidad en la enseñanza de las matemáticas y ciencias. Como fortalezas, entonces, son el incremento en la matrícula, la calidad de la administración de las universidades y los mecanismos de soporte como son el acceso a Internet, la existencia de investigación local y la capacitación de los profesores (López-Leyva, 2020).

\subsection{MÉXICO}

La universidad Privada debe aprender de la Pública, de la investigación, fomento de la ciencia y del Modelo Educativo Integral (Investigación, Docencia, Extensión, Oferta Académica, Internacionalización, Proceso Enseñanza-Aprendizaje, Impacto Social, Vinculación, Filosofía Institucional y Financiamiento). La universidad Pública debe aprender de la Privada, en la SúperEstructura, la visión organizacional adecuada y sólida para su funcionamiento -enfoque comercial-para lograr objetivos institucionales y económicos (Larios-gómez, 2016).

En México, el concepto de responsabilidad social tiene su origen en el ámbito empresarial, para enfatizar aspectos de los procesos productivos ligados al desarrollo social y económico, con criterios de sustentabilidad, es decir tratando de evitar el deterioro del medio ambiente; su transferencia al ámbito de la universidad va cobrando mayor importancia, en la medida en que cada vez un mayor número de instituciones buscan reorientar su desempeño como instituciones socialmente responsables (Cantú, 2021).

\subsection{GUATEMALA}

Dos breves ejemplos de lo anterior pueden observarse en el limitado papel que desarrollaron el Instituto Nacional de Estadística (INE) y la Secretaría Nacional de Ciencia y Tecnología (SENACYT) durante la pandemia en 2020, cuyas naturalezas para las que fueron creadas pudieron haber aportado para que su gestión fuese ordenada, estratégica y eficiente (Velásquez, 2021).

Dentro del Centro Universitario de Zacapa -CUNZAC-, por ser un centro de reciente creación (año 2011), cuenta con carreras que aún no han cumplido un ciclo de cierre de pensum, lo que permite deducir que los profesores de la carrera, son también de reciente incorporación al curso que imparten,...(Rosales, 2021).

\subsection{REPÚBLICA DOMINICANA}

Se concluye que el profesorado de educación superior en las universidades de República Dominicana desarrolla procesos de evaluación del aprendizaje de su alumnado que responden a los fundamentos de la evaluación formativa, pero los modelos de evaluación y acreditación del profesorado 
responden a patrones burocráticos $\mathrm{y}$ administrativos y no a patrones educativos y pedagógicos (González-Barea, Rodríguez-Entrena \& Minyety-Baez, 2021).

La conclusión más significativa, reside en que la participación del profesorado es vital para posibilitar mejores condiciones de vida del entorno, dado que su comportamiento determina las prácticas que las universidades deben adelantar en RSU. Así, se debe apelar a un modelo de gobernanza reticular y no a modelos de estructura vertical que prevalecen en algunas instituciones de educación superior (Rubio-Rodríguez \& Blandón-López, 2021).

\subsection{COSTA RICA}

Hasta 1940, Costa Rica no contaba con universidades, sino con diferentes escuelas de formación profesional”, comentó el Dr. Carlos Araya, de la Universidad de Costa Rica, quien comentó que hoy existen más de 54 universidades públicas y 5 privadas en su país (Palmieri, 2017).

A partir del modelo construido que está mostrando ser efectivo para el producto final que se espera obtener, se han generado una serie de interrogantes sobre cómo mantener continuar manejando un nuevo proceso, a saber, la revisión y la actualización de las políticas de forma que responda a todas las dimensiones de las personas en condición de discapacidad, al cumplimiento de la legislación, a los nuevos contextos que cambian en las universidades y en el país (Palma-Picado, 2017).

\subsection{PUERTO RICO}

En medio de la pandemia, la universidad ha operando ininterrumpidamente mediante herramientas digitales, con más de 11,400 cursos en línea. Al momento, la administración universitaria gestiona un aumento en su programas académicos a distancia, tras lograr nueva reglamentación para su aprobación en un tiempo de evaluación menor. La institución tiene bajo evaluación unos 35 reglamentos y políticas institucionales para agilizar procesos académicos y de gobernanza (UPR, 2020).

La abarcadora radiografía de las universidades públicas y privadas de Puerto Rico, a juicio de Excelencia in Education, puede ser aleccionadora para las instituciones universitarias de Estados Unidos. La investigación destaca la resiliencia de las instituciones universitarias en la Isla, tras enfrentar huracanes, terremotos y crisis económicas (FPHPR, 2020).

\subsection{HONDURAS}

Con el apoyo técnico y financiero de la Agencia Suiza para el Desarrollo y la Cooperación (COSUDE), y una contrapartida de la Universidad Zamorano, inició la implementación del proyecto "Fortalecimiento de la Gestión Universitaria frente al Cambio Climático y la Reducción de Riesgo ante Desastres." Este proyecto busca fortalecer las capacidades de las 21 universidades de Honduras que 
conforman el Comité Interinstitucional de Ciencias Ambientales (CICA) mediante procesos de enseñanza e investigación en Adaptación Frente al Cambio Climático (ACC) y la Reducción de Riesgos de Desastres (RRD) de origen climático, socioeconómico y epidemiológico (Zamorano, 2021).

El rector de la Universidad Nacional Autónoma de Honduras (UNAH) y presidente del Consejo de Educación Superior (CES), Francisco J. Herrera Alvarado, informó que remitirán al Sistema Nacional de Gestión de Riesgos (Sinager) una solicitud de autorización para el retorno progresivo a la semipresencialidad en las diferentes universidades públicas y privadas del país (Red Comunica, 2021).

\subsection{EL SALVADOR}

El Rector de la Universidad de El Salvador informa a la nación que: Esta casa de estudios ha sido acreditada por la Agencia de Autoevaluación y Acreditación de la Educación Superior de Francia (HCERES), reconocimiento que CERTIFICA la calidad de nuestros procesos académicos, científicos y administrativos. De esta forma honramos y rendimos cuentas al pueblo salvadoreño de nuestra excelencia académica y compromise (Universidad de El Salvador, 2021).

Docentes de esta casa de estudios culminaron el postgrado en Campus Virtuales para la Práctica Educativa y recibieron sus diplomas de aprobación en un acto que reunió a autoridades académicas de la institución. Con este postgrado los docentes Utec fortalecen sus competencias en el área de educación virtual, metodología en la que la esta casa de estudios es pionera en el país (Universidad Tecnológica de El Salvador, 2021).

\subsection{NICARAGUA}

Nicaragua, cuenta con dos instancias: el Consejo Nacional de Universidades (CNU) como ente rector, cuyo "n es, el apoyo y asesoría a la educación superior; instituido el , de abril de \%--\&, y el Consejo Nacional de Evaluación y Acreditación (CNEA), creado en el $+\& \% \%$ mediante la ley *\&(; quien es la máxima autoridad del Sistema Nacional para el aseguramiento de la calidad (HernándezCastellón \& Zamora-Díaz, 2020).

Es a raíz de este análisis que se retoma el Plan Nacional de Desarrollo Humano, asumido por el Gobierno de Reconciliación y Unidad Nacional, entre 2018-2021; el cual contempla la Educación en todas sus formas, como un eje medular del desarrollo social, desde lo local y nacional, mediante estrategias creativas, innovadoras y alternantes entre el campo formal y no formal (Ruiz, López, López, Pérez \& Matus, 2021). 


\subsection{PANAMÁ}

El proceso de acreditación institucional panameño tuvo mucha relevancia a nivel internacional, ya que marcó la diferencia con otros países, al ser obligatorio para todas las universidades tanto oficiales como particulares que operasen en el territorio nacional (Álvarez de Barrios, Castillero \& Sousa, 2020).

De los resultados obtenidos emanó un modelo que se complementa con las características de las editoriales universitarias, aunque aún hace falta realizar el cambio que las impulse a la creación de una red nacional de alianzas, que promocione los productos y servicios, y otorgue el apoyo necesario al usuario (Batista de Muñoz, 2021).

\subsection{CUBA}

La aplicación del procedimiento al proceso de Planificación Estratégica en la Universidad de Cienfuegos, permitió: Ubicar el proceso dentro del mapa de procesos de la organización constatándose que forma parte de los procesos directrices de la organización, describer el proceso, estableciendo entradas, salidas, proveedores, usuarios y sus actividades, evaluar el proceso determinado los principales problemas del mismo (Alonzo, 2016).

Entre los desafíos de la Universidad de Ciencias Médicas de Granma (UCMG) está la formación de profesionales competentes y también de ciudadanos comprometidos con la sociedad, tanto en los niveles de pregrado como de postgrado, esto requiere de cambios en la cultura organizacional del trabajo metodológico que permita elevar la calidad del proceso enseñanza -aprendizaje...(Martínez, Ramos \& Salgado, 2020).

\subsection{COLOMBIA}

Los resultados permiten evidenciar que las universidades no solo han adaptado su gestión para continuar con sus funciones misionales (docencia, investigación y extensión), sino que también han asumido un rol protagónico para contribuir a la gestión en la pandemia en el país gracias a sus conocimientos, capital humano, desarrollo tecnológico y científico, y capacidades de infraestructura, laboratorios y tecnología (Bedoya-Dorado, Murillo-Vargas \& González-Campo, 2021).

La muestra fue de 214 estudiantes, pertenecientes a diversos programas de la Corporación Universitaria Autónoma de Nariño...En este sentido, se formuló un modelo que facilite la gestión universitaria de la innovación social en la institución objeto de estudio, que articula las dependencias de emprendimiento, investigación y proyección social, para que los beneficios se orienten tanto a la comunidad universitaria como a los territories (Jurado-Paz \& Morán-Vallejo, 2019). 


\subsection{VENEZUELA}

En las IES situadas en la ciudad de Maracaibo, Estado de Zulia, en Venezuela: Universidad Rafael Belloso Chacín (URBC), Universidad Rafael Urdaneta (URU) y la Universidad José Gregorio Hernández,... se observó que en las IES se deben aplicar modelos de gestión del conocimiento, con el fin de generar, transformar, integrar y difundir el conocimiento, de este modo, generar aprendizaje significativo mediante la producción intelectual y el uso de las TIC como medio de transmisión interactivo,...(Milla, Martelo \& Peña, 2018).

Como universidad médica inmersa en la generación de modelos de formación que garanticen egresados transformadores de los problemas sociales, ha contribuido -a consideración de los autores de este artículo- con la Educación Médica en lo siguiente: enseñanza en el servicio asistencial y educación en el colectivo laboral, desarrollo de nuevas formas de organización de la enseñanza,...(Milián \& Romero, 2020).

\subsection{ECUADOR}

La aplicación durante tres años del Modelo de la Pirámide de Desarrollo Universitario en la gestión

estratégica Universidad de Otavalo contribuyó a mejorar los resultados de la institución en las áreas de claustro, formación académica, investigación, vinculación con la sociedad, aseguramiento logístico y en su conjunto posibilitó obtener la condición de universidad acreditada, nunca antes alcanzada por la institución, con lo que se comprobó la hipótesis planteada en la investigación (Romillo y Polaino, 2019).

La investigación se realiza en el contexto de la universidad ecuatoriana en tiempos de avance de la IV Revolución industrial. Se realiza el estudio desde las elaboraciones que aportan el pensamiento complejo y el enfoque multidimensional sistémico. Se develan la importancia de asumir la gestión del talento humano en las diferentes fases de la planeación universitaria, y en interrelación con las triangulaciones que la gestión del conocimiento incorpora al desempeño de las funciones sustantivas universitarias (Briones \& González, 2019).

\subsection{PERÚ}

Se presenta una investigación que analiza las estrategias de gestión del capital humano, con respecto a los docentes, en la Facultad de Educación de una universidad de Lima... se obtuvo información sobre las estrategias dirigidas a valorar y potenciar el capital humano en la institución. Aunque los resultados evidenciaron que estas son insuficientes, se destaca el compromiso institucional de los docents (Cuentas, 2018). 
Se concluye con el diseño de un nuevo modelo de gestión, basado en tres pilares: institucionalidad, para garantizar la adopción del modelo en la universidad; gestión de la investigación propiamente dicha, que busca fortalecer las competencias para la publicación de artículos científicos; y gestión administrativa, para asegurar las condiciones logísticas y operativas en la ejecución de los proyectos de investigación (Valles-Coral, 2019).

\subsection{BRASIL}

Se trabaja con el objetivo de sistematizar las experiencias de coopetición entre las Instituciones de Educación Superior del Consorcio de Universidades Comunitarias del Estado de Rio Grande do Sul, Brasil (COMUNG) a través de la participación en actividades que promuevan la gestión sostenible... Concluimos por la coopetición como una estrategia asertiva y potencializadora del empoderamiento del COMUNG para enfrentar sus desafíos, especialmente en tiempos de COVID-19 (Susana, de Quadros, Vaz \& Fossatti, 2021).

A pesar del corto tiempo en el que CSF funcionó, tuvo un impacto importante en aumentar la presencia brasileña en el exterior y en crear un ambiente internacional en las universidades más prestigiosas, aunque fue muy criticado por el modo en que se asignaron recursos para su financiamiento la falta de retorno institucional. Sin dudas, marcó un antes y un después en los procesos de internacionalización académica en Brasil (Perrotta y Sharpe, 2020).

\subsection{BOLIVIA}

Aun cuando el estudio identificó que las medidas legislativas analizadas orientan sus acciones a alcanzar la dimensión de aceptabilidad, se muestra que el Estado boliviano tiene obligaciones centrales en materia educativa, así por ejemplo los criterios de accesibilidad y permanencia están enunciados en la Constitución Política del Estado como la Ley 070; sin embargo, no se hacen efectivas dentro de las políticas o declaraciones objetivas para la Formación Superior Universitaria (Pinto, 2021).

Las revistas de la materia de Ciencias de la Salud se orientan hacia los objetivos de SciELO Bolivia, el mismo que llegará a alcanzarse principalmente con el trabajo conjunto de autores, universidades y hospitals (Claros \& Claros, 2020).

\subsection{URUGUAY}

El propósito del presente artículo es explorar la gradual emergencia de un campo de investigación en educación superior en Uruguay entre 1990 y 2020...La institucionalización del mismo se expresa en factores institucionales y disciplinarios. Dicho campo de investigación aparece aún en proceso de 
consolidación, promovido a la vez por desafíos de las políticas públicas a nivel local, tanto como por procesos regionales y externos (Martínez \& Chiancone, 2021).

Durante los gobiernos progresistas en Uruguay (2005-2019) se desarrollaron procesos de “privatización latente”. Estos fueron promovidos por sectores de derecha y tuvieron condiciones para su consolidación debido a la ausencia de debate político-pedagógico por parte de la izquierda. Ello puede deberse a que las fuerzas progresistas asumieron una visión consensual de la democracia, rehuyendo su carácter antagónico. Los procesos de privatización articularon modalidades exógenas y endógenas, con una primacía de estas últimas (Martinis, 2020).

\subsection{PARAGUAY}

La gestión y reducción de riesgo de desastres se plantea como desafío para las universidades,... resaltan como buenas prácticas: los talleres sobre temas relacionados con la gestión de riesgos de desastres, la evaluación de la seguridad de la institución, la convocatoria a actores de diferentes instituciones en las actividades de capacitación y la inclusión en el proyecto de acciones que afectan a la formación e investigación (Zavala, Backes, Ríos \& Colmán, 2021).

Los resultados obtenidos indican que, la gestión estratégica de los Centros de Investigación, necesitan potenciar los aspectos mencionados para obtener mejores resultados como ser, aumentar la producción científica, mayor presupuesto, mejor gerenciamiento en todos los centros de Investigación de la Universidad Nacional de Asunción (UNA); así como mayor visibilidad a nivel institucional de los mismos (Aguilar-de Cáceres, 2020).

\subsection{ARGENTINA}

Queremos finalmente subrayar que nuestros juicios no pretenden llevar a cabo un cuestionamiento de raíz a la configuración de un sistema universitario masivo y abierto basado en la gratuidad y el ingreso irrestricto. Particularmente hemos querido subrayar sus limitaciones en el intento de avanzar en una educación más democrática, inclusiva y de calidad (Buchbinder, 2020).

Es probable también que, con el sustancial desarrollo producido en el sistema universitario argentino y el actual contexto organizacional, se deban contemplar nuevas políticas de gestión institucional que posibiliten a las universidades adaptarse a nuevas demandas y exigencias, con el objeto de preservar e incrementar los estándares de calidad (Antonio-Castillo \& Ganga-Contreras, 2021). 


\subsection{CHILE}

En el contexto chileno, a tales desafíos mundiales de la educación superior, se añaden factores como la expansión del acceso, la proliferación de nuevos programas o la emergencia de universidades más complejas-, que incrementan la complejidad e incertidumbre del entorno (Cisternas, 2021).

Según lo descrito en esta investigación, lograr mejoras en la calidad de los servicios entregados por la Universidad será posible solo si se entiende y valora los procesos de gestión de calidad como principios cardinales donde todos los que forman parte de la institución son corresponsables y además, están dispuestos al diálogo necesario para establecer una caracterización de que la institución entenderá por calidad, considerando las perspectivas de todos sus actores, especialmente, la de sus estudiantes (Ruff, Ruiz, Matheu, Juica \& Anabalón, 2021).

Las universidades latinoamericanas, se han visto forzadas por la pandemia a migrar a la educación virtual, con algunas dificultades como la falta de equipos tecnológicos, acceso a Internet y capacitación del personal. Además, están optimizando su modelo de gestión universitaria.

\section{CONCLUSIONES}

En las universidades latinoamericanas, los factores con mayor influencia en la gestión universitaria actual son la infraestructura, la gestión de la información, inversión en sistemas de información, capacitación de los recursos humanos, empleabilidad del egresado, calidad de los procesos académicos, científicos y administrativos, responsabilidad social, competencias en el área de educación virtual, rol protagónico en la gestión de la pandemia, gestión de riesgos de desastres y el regreso a la presencialidad. 


\section{REFERENCIAS}

Aguilar-de Cáceres, Mabel Carolina. (2020). Gestión estratégica en los centros de investigación de la Universidad Nacional de Asunción. Población y Desarrollo, 26(51), 20-25. Epub December 00, 2020.https://dx.doi.org/10.18004/pdfce/2076-054x/2020.026.51.020

Alonzo, K. R. (2016). El enfoque de procesos en la planificación estratégica en la universidad de cienfuegos. Estrategia y Gestión Universitaria, 4(2).

Antonio-Castillo, Juan, \& Ganga-Contreras, Francisco (2021). Gobernanza universitaria: análisis de situación desde la perspectiva de la Ley de Educación Superior Argentina. Revista Cubana de Educación Superior, 40(2), e18. Epub 01 de abril de 2021. Recuperado de http://scielo.sld.cu/scielo.php?script=sci_arttext\&pid=S0257-43142021000200018\&lng=es\&tlng=es.

Álvarez de Barrios, A. M., Castillero Britton, Y., \& Sousa Valdés, V. (2020). Evaluación a nivel Nacional e Internacional de la Acreditación Superior Universitaria en Panamá. Revista Conrado, 16(75), 225-230.

Banco Mundial (2021). América Latina y el Caribe: panorama general. Recuperado de https://www.bancomundial.org/es/region/lac/overview

Barrero, A. y Rosero, A. (2018). Estado del Arte sobre Concepciones de la Diversidad en el Contexto Escolar Infantil. Revista Latinoamericana de Educación Inclusiva, 2018, 12(1), 39-55 https://doi.org/10.4067/S071873782018000100004

Barquero-Cabrero, J.-D., Barceló-Sánchez, J.-M., López-Martín, J.-A., \& Cabezuelo-Lorenzo, F. (2020). Gestión universitaria ante el virus Covid-19: análisis de un caso español. Revista Venezolana De Gerencia, 25(91), 1126-1139. https://doi.org/10.37960/rvg.v25i91.33186

Basile, Gonzalo (2020). SARS-CoV-2 en América Latina y Caribe: Las tres encrucijadas para el pensamiento crítico en salud. Ciência \& Saúde Coletiva [online]. v. 25, n. 9, pp. 3557-3562. Disponible en: <https://doi.org/10.1590/1413-81232020259.20952020>. ISSN 1678-4561. https://doi.org/10.1590/141381232020259.20952020 .

Batista de Muñoz, L. (2021). Gestión de editoriales universitarias y la transferencia estratégica de conocimiento en Panamá. Vol. 13 Núm. 2 (2021): SIGNOS, investigación en sistemas de gestión. https://doi.org/10.15332/24631140.6665

Bedoya-Dorado, Cristian, Murillo-Vargas, Guillermo, \& González-Campo, Carlos Hernán. (2021). Gestión universitaria en tiempos de pandemia por COVID-19: análisis del sector de la educación superior en Colombia. Estudios Gerenciales, 37(159), 251-264. Epub July 27, 2021.https://doi.org/10.18046/j.estger.2021.159.4409

Briones Cedeño, Karen Lorena, \& González Calzadilla, Claribel Silvia. (2019). La universidad contemporánea y la gestión del talento humano. Apuntes y reflexiones. Revista San Gregorio, (35), 81-93. https://doi.org/10.36097/rsan.v1i35.1191

Buchbinder, Pablo. (2020). El sistema universitario argentino: una lectura de sus transformaciones en el largo plazo (1983-2015). Revista de la educación superior, 49(193), 45-64. Epub 21 de agosto de 2020. https://doi.org/10.36857/resu.2020.193.1026 
Cantú Mendoza, R. (2021). La relevancia de la educación superior y calidad de vida: pensar la comunidad en el marco de la agenda 2030. Transdisciplinar Revista De Ciencias Sociales Del CEH, 1(1). https://doi.org/10.29105/transdisciplinar1.1-6

Claros Coca, Z., \& Claros Coca, E. (2020). Producción del área de Ciencias de la Salud - SciElO Bolivia, gestión 2009-2017. Gaceta Médica Boliviana, 41(1), 14-19. https://doi.org/10.47993/gmb.v41i1.144

CEPAL (2021a). El Foro de los Países de América Latina y el Caribe sobre el Desarrollo Sostenible y el Seguimiento Regional de la Agenda 2030. Recuperado de https://agenda2030lac.org/es/el-foro-de-los-paisesde-america-latina-y-el-caribe-sobre-el-desarrollo-sostenible-y-el-seguimiento

CEPAL (2021b). En América Latina y el Caribe: el nuevo informe de la ONU advierte sobre una recuperación económica frágil e irregular. Recuperado de https://www.cepal.org/es/comunicados/america-latina-caribenuevo-informe-la-onu-advierte-recuperacion-economica-fragil

Cisternas, C. (2021). Análisis del Entorno en la Gestión Universitaria: Una Aproximación desde la Teoría de Sistemas Sociales. Actualidades Investigativas en Educación, vol. 21, núm. 1, pp. 457-479. https://doi.org/10.15517/aie.v21i1.44075

Corral, C., Izurieta, L. \& Macías, M. (2020). Gestión universitaria en post-pandemia: implicaciones para una estrategia de gobierno electrónico. Revista Educare UPEL-IPB Barquisimeto, Edo. Lara - Venezuela. Vol. $24 \mathrm{~N}^{\circ}$ 3. Septiembre - Diciembre 2020. https://doi.org/10.46498/reduipb.v24i3.1403

Cuentas Martel, Susan Carol. (2018). La gestión del capital humano en una unidad académica de educación superior: analysis of a university. Educación, 27(53), 57-72. https://dx.doi.org/10.18800/educacion.201802.004

FPHPR (2020). Nueva investigación proporciona contexto para la Resiliencia Institucional en Puerto Rico. Recuperado de https://fphpr.org/nueva-investigacion-proporciona-contexto-para-la-resiliencia-institucionalen-puerto-rico/

Gerón-Piñón, Gabriela, Solana-González, Pedro, Trigueros-Preciado, Sara, \& Pérez-González, Daniel. (2021). Sistemas de información en las universidades latinoamericanas: su impacto en los rankings internacionales. Revista de la educación superior, 50(198), 23-35. Epub 11 de octubre de 2021.https://doi.org/10.36857/resu.2021.198.1699

Gil Álvarez, J. L., Morales Cruz, M., \& Nieto Almeida, L. E. (2020). La gestión universitaria en tiempos de Covid-19: Retos, nuevas prácticas y aprendizajes. Revista Metropolitana de Ciencias Aplicadas, 3(3), 270277

González-Barea, Eva M., Rodríguez-Entrena, María J., \& Minyety-Baez, María E.. (2021). Evaluación de la educación universitaria en República Dominicana. Formación universitaria, 14(2), 13-24. https://dx.doi.org/10.4067/S0718-50062021000200013

Hernández-Castellón, J. A., \& Zamora-Díaz, W. J. (2020). Sistemas de gestión de la calidad: Una mejora en la calidad de las Instituciones de Educación Superior en Nicaragua. Revista Electrónica de Conocimientos, Saberes y Prácticas, 3(2), 87-97. https://doi.org/10.5377/recsp.v3i2.10694

Jurado-Paz, I. M., \& Morán-Vallejo, M. A. (2019). Gestión universitaria de la innovación social promovida desde espacios académicos relacionados con el emprendimiento, la investigación y la proyección social. Revista de Investigación, Desarrollo e Innovación, 9(2), 261-272. https://doi.org/10.19053/20278306.v9.n2.2019.9161 
Larios-gómez, Emigdio. (2016). LA GESTIÓN EDUCATIVA PARA LA UNIVERSIDAD PÚBLICA Y PRIVADA EN MÉXICO: UNA COMPARACIÓN COMPETITIVA. Recuperado de https://www.researchgate.net/publication/338750487_LA_GESTION_EDUCATIVA_PARA_LA_UNIVER SIDAD_PUBLICA_Y_PRIVADA_EN_MEXICO_UNA_COMPARACION_COMPETITIVA

López-Leyva, Santos. (2020). Fortalezas y debilidades de la educación superior en América Latina para la competitividad global. Formación universitaria, 13(5), 165-176. https://dx.doi.org/10.4067/S071850062020000500165

Marcelo, Carlos y Vaillant, Denise (2017). Políticas y programas de inducción en la docencia en Latinoamérica. Cadernos de Pesquisa. v. 47, n. 166, pp. 1224-1249. Disponible en: <https://doi.org/10.1590/198053144322>. ISSN 1980-5314. https://doi.org/10.1590/198053144322.

Martínez Barreiro., Luis Armando, Ramos Socarras., Anival Ernesto, \& Salgado Selema., Guillermo. (2020). Pilares estratégicos del sistema de gestión universitaria en el curso 2019 -2020. Multimed, 24(4), 969-978. Epub 10 de julio de 2020. Recuperado en 27 de noviembre de 2021, de http://scielo.sld.cu/scielo.php?script=sci_arttext\&pid=S1028-48182020000400969\&lng=es\&tlng=es

Martínez Larrechea, E., \& Chiancone, A. (2021). La construcción del campo de investigación sobre educación superior en Uruguay: desafíos políticos e investigación. Revista Educación Superior Y Sociedad (ESS), 33(1), 235-261. Recuperado a partir de https://www.iesalc.unesco.org/ess/index.php/ess3/article/view/363

Martinis, Pablo (2020). Disputas en torno al carácter de la educación pública en Uruguay: de la privatización latente a la privatización impuesta. Educar em Revista. v. 36, e77559. Disponible en: $<$ https://doi.org/10.1590/0104-4060.77559>. Epub 21 Dic 2020. ISSN 1984-0411. https://doi.org/10.1590/0104-4060.77559.

Milián Vázquez, Pedro Miguel, \& Romero Sánchez, María del Carmen. (2020). Contribuciones de la Universidad de las Ciencias de la Salud en Venezuela a la Educación Médica. MediSur, 18(6), 1246-1249. Epub 02 de diciembre de 2020. Recuperado en 01 de diciembre de 2021, de http://scielo.sld.cu/scielo.php?script=sci_arttext\&pid=S1727-897X2020000601246\&lng=es\&tlng=es.

Milla Calderon, L. E., Martelo Gómez, R. J., \& Peña Pertuz, M. (2018). Gestión del conocimiento para la difusión de producción intelectual en la educación universitaria. Saber, Ciencia Y Libertad, 13(1), 290-303. https://doi.org/10.18041/2382-3240/saber.2018v13n1.2569

Murillo, M (2019). Gestión universitaria. Concepto y principales tendencias. Revista Atlante: Cuadernos de Educación y Desarrollo (marzo 2019). Recuperado de https://www.eumed.net/rev/atlante/2019/03/gestion-universitaria.html //hdl.handle.net/20.500.11763/atlante1903gestion-universitaria

Naciones Unidas (2020). La ONU ayuda a América Latina para frenar urgentemente la propagación del COVID-19. Recuperado de https://www.un.org/es/coronavirus/articles/onu-apoya-america-latina-contrapropagacion-covid-19

Oficina Universitaria de Imagen Institucional (2019). UNSA presente en el XIX Coloquio Internacional de Gestión Universitaria - CIGU 2019. Recuperado de https://www.unsa.edu.pe/unsa-presente-en-el-xixcoloquio-internacional-de-gestion-universitaria-cigu-2019/

Palma-Picado, K. (Agosto, 2017). Gestión de políticas universitarias para personas con discapacidad en el Instituto Tecnológico de Costa Rica: un modelo para el proceso de construcción. Trabajo presentado en el Primer Encuentro de Centroamérica y México sobre Investigación y Discapacidad en la Educación 
Superior. Ciudad de Guatemala, Guatemala. Recuperado de https://repositoriotec.tec.ac.cr/bitstream/handle/2238/10478/DOP_PON_12_gestiondiscapacidad_2017.pdf?sequence=1\&isAllowed=y

Palmieri, F. (2017). Gestión universitaria del siglo XXI. Recuperado de https://puntoedu.pucp.edu.pe/noticia/gestion-universitaria-del-siglo-xxi/

Perrotta, Daniela y Sharpe, Andrés Santos (2020). Política exterior y procesos de internacionalización del sistema científico y universitario: Argentina y Brasil (2003-2019). Sociologias [online]. 2020, v. 22, n. 54, pp. 88-119. Disponible en: <https://doi.org/10.1590/15174522-99655>. Epub 31 Ago 2020. ISSN 1807-0337. https://doi.org/10.1590/15174522-99655.

Pinto López, Rubén Alberto. (2021). ¿Quiénes son los menos aventajados?: desigualdad educativa en la educación superior. Revista Ciencia y Cultura, 25(46), 111-133. Recuperado en 01 de diciembre de 2021, de http://www.scielo.org.bo/scielo.php?script=sci_arttext\&pid=S2077-33232021000100006\&lng=es\&tlng=es.

Red Comunica (2021). Consejo de Educación Superior solicitará a Sinager autorización para el retorno progresivo a la semipresencialidad. Recuperado de https://redcomunica.csuca.org/index.php/universidadnacional-autonoma-de-honduras-unah/consejo-de-educacion-superior-solicitara-a-sinager-autorizacionpara-el-retorno-progresivo-a-la-semipresencialidad/

Romillo, A. y Polaino, C. (2019). Aplicación del Modelo de Gestión Pirámide del Desarrollo Universitario en la Universidad de Otavalo, Ecuador. Formación Universitaria. Vol. 12(1), 3-12 (2019). http://dx.doi.org/10.4067/S0718-50062019000100003

Roque Paredes, O. (2018). La Gestión Universitaria y la empleabilidad del egresado de la Facultad de Arquitectura de la Universidad Ricardo Palma. Tradición, Segunda época, (17), 113 - 123. https://doi.org/10.31381/tradicion.v0i17.1373

Rosales Gómez, M. A. (2021). Competencias del profesor de la carrera de Administración de Empresas del Centro Universitario de Zacapa -CUNZAC-. Revista Ciencia Multidisciplinaria CUNORI, 5(1), 51-64. https://doi.org/10.36314/cunori.v5i1.152

Rubio-Rodríguez, Gustavo A., \& Blandón-López, Alexander. (2021). El profesorado y la responsabilidad social universitaria: un análisis cualitativo de redes. Formación universitaria, 14(2), 3-12. https://dx.doi.org/10.4067/S0718-50062021000200003

Ruiz Chow, L. C. A. ., López Carcache, A. ., López Vásquez, R. A. ., Pérez Mendoza, L. E., \& Matus Rodríguez, F. E. (2021). Estrategias Educativas Emergentes para el fortalecimiento de la educación superior en Nicaragua: tensiones y perspectivas. Revista Compromiso Social, (5), 120-130. Recuperado a partir de https://revistacompromisosocial.unan.edu.ni/index.php/CompromisoSocial/article/view/113

Ruff Escobar, C., Ruiz Toledo, M., Matheu Pérez, A., Juica Martínez, P., \& Anabalón Arenas, G. (2021). Efectividad de la gestión en las universidades, desde modelos de percepción de calidad de estudiantes: el modelo de la Universidad Bernardo O'Higgins. Revista De Métodos Cuantitativos Para La Economía Y La Empresa, 31, 259-279. https://doi.org/10.46661/revmetodoscuanteconempresa.4336

Statista Research Department (2021). América Latina y el Caribe - Datos estadísticos. Recuperado de https://es.statista.com/temas/5605/america-latina-y-el-caribe/\#dossierKeyfigures 
Susana jung, H., de Quadros da Silva, L., Vaz, D., \& Fossatti, P. (2021). Coopetición en la gestión académica de la educación superior en Rio Grande Do Sul, Brasil . Journal of the Academy, (5), 78-98. https://doi.org/10.47058/joa5.6

Tezanos, S. (2018). Geografía del desarrollo en América Latina y el Caribe: hacia una nueva taxonomía multidimensional de los Objetivos de Desarrollo Sostenible. Recuperado de https://www.cepal.org/es/publicaciones/43989-geografia-desarrollo-america-latina-caribe-nueva-taxonomiamultidimensional

UNESCO (2021). Educación superior. Recuperado de https://es.unesco.org/themes/educacion-superior

Universidad de El Salvado (2021). Universidad Católica de El Salvador - UNICAES. Recuperado de https://www.ues.edu.sv/

Universidad Tecnológica de El Salvador (2021). Graduación del postgrado en Campus Virtuales para la Práctica Educativa. Recuperado de https://www.utec.edu.sv/Inicio/Noticias/graduacion-del-postgrado-encampus-virtuales-para-la-practica-educativa-

UPR (2020). Presidente de la UPR presenta resultados de su gestión administrativa y solicita detener reducciones al presupuesto institucional. Recuperado de https://www.upr.edu/presidente-de-la-upr-presentaresultados-de-su-gestion-administrativa-y-solicita-detener-reducciones-al-presupuesto-institucional/

Valles-Coral, M. A. (2019). Modelo de gestión de la investigación para incrementar la producción científica de los docentes universitarios del Perú. Revista de Investigación, Desarrollo e Innovación, 10(1), 67-78. https://doi.org/10.19053/20278306.v10.n1.2019.10012

Velásquez Pérez, L. (2021). Asesoría científica y política pública: La gestión de la pandemia en Guatemala. Política. Revista de Ciencia Política, 59(1), 11-33. doi:10.5354/0719-5338.2021.61104

Veliz-Briones, Vicente Felix, Alonso-Becerra, Alicia, Fleitas-Triana, María Sonia, \& Alfonso-Robaina, Daniel. (2016). Una gestión universitaria basada en los enfoques de gestión de proyecto y por proceso. Revista Electrónica Educare, 20(3), 466-482. https://dx.doi.org/10.15359/ree.20-3.23

Zamorano (2021). Zamorano, Suiza y el CICA facilitan la gestión ambiental de 21 universidades hondureñas. Recuperado de https://www.zamorano.edu/2021/04/21/zamorano-suiza-y-el-cica-facilitan-la-gestionambiental-de-21-universidades-hondurenas/

Zavala Saucedo, M. V. ., Backes Dos Santos, K. N. ., Ríos Zavala, S. M., \& Colmán, D. S. (2021). Gestión de riesgo de desastres en instituciones de educación superior : una mirada desde la aplicación de un proyecto. Investigaciones Y Estudios - UNA, 12(1), 58-63. Recuperado de https://revistascientificas.una.py/index.php/rdgic/article/view/972 